\title{
Abnormal splicing of hepatocyte nuclear factor-1 beta in the renal cysts and diabetes syndrome
}

\author{
L. W. Harries ${ }^{1}$ S. Ellard ${ }^{1,3}$ • R. W. A. Jones ${ }^{2}$ A. T. Hattersley ${ }^{1}$ C. Bingham ${ }^{1}$ \\ ${ }^{1}$ Molecular Genetics, Institute of Biomedical and Clinical Science, Peninsula Medical School, Exeter, UK \\ 2 Department of Child Health, Derriford Hospital, Plymouth, UK \\ ${ }^{3}$ Molecular Genetics, Royal Devon and Exeter Hospital (Wonford), Exeter, Devon, UK
}

\section{Abstract}

Aims/hypothesis. Mutations in the hepatocyte nuclear factor-1 beta $(H N F-1 \beta)$ gene result in disorders of renal development, typically involving renal cysts and early-onset diabetes (the RCAD syndrome/ MODY5). Sixteen mutations have been reported, including three splicing mutations of the intron 2 splice donor site. Because tissues showing abundant expression (kidney, liver, pancreas, gut, lung and gonads) are not easily accessible for analysis in living subjects, it has previously proven difficult to determine the effect of $H N F$ $1 \beta$ mutations at the mRNA level. This is the aim of the present study.

Methods. We have developed a nested RT-PCR assay that exploits the presence of ectopic $H N F-1 \beta$ transcripts in Epstein-Barr virus (EBV)-transformed lymphoblastoid cell lines derived from subjects carrying $H N F-1 \beta$ splice site mutations.
Results. We report a fourth mutation of the intron 2 splice donor site, IVS2nt+2insT. Sequence analysis of ectopic $H N F-1 \beta$ transcripts showed that both IVS2nt+2insT and IVS2nt $+1 \mathrm{G}>\mathrm{T}$ result in the deletion of exon 2 and are predicted to result in premature termination of the HNF-1 $\beta$ protein. Mutant transcripts were less abundant than the normal transcripts but there was no evidence of nonsense-mediated decay.

Conclusions/interpretation. This is the first study to define the pathogenic consequences of mutations within the $H N F-1 \beta$ gene by mRNA analysis. This type of approach is a useful and important tool to define mutational mechanisms and determine pathogenicity.

Keywords HNF- $1 \beta$ - Illegitimate transcription • MODY5 $\cdot$ RCAD $\cdot$ RT-PCR $\cdot$ Splicing.
Received: 3 October 2003 / Accepted: 15 January 2004

Published online: 15 April 2004

C) Springer-Verlag 2004

S. Ellard $(\bowtie)$

Molecular Genetics,

Royal Devon and Exeter Hospital (Wonford),

Barrack Road, Exeter, Devon, EX2 5DW, UK

E-mail: S.Ellard@exeter.ac.uk

Tel.: +44-1392-402901, Fax: +44-1392-402903

Abbreviations: $\mathrm{Ct}$, crossing point - EBV, Epstein-Barr virus · HNF-1 $\beta$, hepatocyte nuclear factor beta - MODY5, maturity-onset diabetes of the young subtype 5 .

NMD, nonsense-mediated decay $\cdot$ RCAD, renal cysts and diabetes syndrome

\section{Introduction}

The renal cysts and diabetes (RCAD) syndrome is associated with heterozygous mutations in the hepatocyte nuclear factor-1 beta $(H N F-1 \beta)$ gene $[1,2,3,4,5]$. RCAD has an autosomal dominant pattern of inheritance, although spontaneous mutations have been described $[1,2,3,4,5,6]$. The RCAD phenotype is variable, with renal disease (particularly renal cystic disease) being the most consistent feature. A variety of renal histologies have been reported, but all are the result of abnormal renal development $[3,4,5,6,7,8]$. Some subjects have diabetes, which typically develops after the onset of renal disease. The age of diagnosis ranges from 12 to 61 years [4]. Genital tract malformations, abnormal liver function, hyperuricaemia and gout are 
also present in some kindreds $[4,7,9,10,11]$. Diabetes in these kindreds is classified as maturity-onset diabetes of the young subtype 5 (MODY5) [12]. HNF-1 $\beta$ is expressed in the kidney, pancreas, liver, gonads, gut, lung and thymus. The phenotypes produced by mutations in the $H N F-1 \beta$ gene are complex and variability is evident both between and within families. All families with $H N F-1 \beta$ mutations exhibit some form of renal phenotype, whereas the diabetes is more variable and incompletely penetrant (Fig. 1). This may reflect differences in temporal or spatial expression patterns between renal and pancreatic tissues.

Sixteen $H N F-1 \beta$ mutations have been reported since the initial description in 1997 [1]. These include insertion/deletion mutations, missense mutations, nonsense mutations and splice site mutations $[1,2,3,4,5$, $6,7,8,9,10,11,13,14,15]$.

Mutations that affect mRNA processing can result in an altered reading frame, with the production of an mRNA transcript harbouring a premature termination codon. The consequences of premature termination (the production of dominant-negative or inactive truncated proteins) are potentially harmful to the cell. Both prokaryotic and eukaryotic systems have therefore evolved pathways that detect and degrade such transcripts. One such pathway is the nonsense-mediated decay (NMD) mRNA surveillance pathway [16, 17]. This is a mechanism by which a complex of proteins deposited by the spliceosome during mRNA processing interacts with a stalled ribosome at the site of the premature termination codon and causes the polyadenylation-independent decapping and subsequent degradation of the mRNA.

Three splicing mutations have been detected at the site of a putative mutation hotspot within the splice donor site of intron $2[9,11,14]$. These mutations are IVS2nt $+1 \mathrm{G}>\mathrm{T}$, IVS2nt+1G $>\mathrm{A}$ and IVS2nt+2delAAGT. Since tissues with high native HNF-1 $\beta$ expression are not accessible, it has not been possible to investigate the effect of these splice site mutations on the stability and processing of $H N F-1 \beta$ mRNA. Illegitimate or ectopic transcription (the presence of very low quantities of correctly spliced tissue-specific mRNAs in non-expressing tissues) allows the examination of transcripts in transformed lymphocytes. We have recently reported an assay that used the presence of ectopic transcripts within lymphoblastoid cells to examine the effect of three splice site mutations of the HNF-l $\alpha$ gene [18]. This approach has also been used to examine the effects of a splice site mutation in the glucokinase $(G C K)$ gene [19].

We have now developed a similar two-round nested RT-PCR assay that allows the amplification of $H N F-1 \beta$ mRNA transcripts from lymphoblastoid cell lines. This approach has been used to determine the mutational mechanisms of two splice site mutations in the $H N F-1 \beta$ gene from kindreds with the RCAD syndrome.
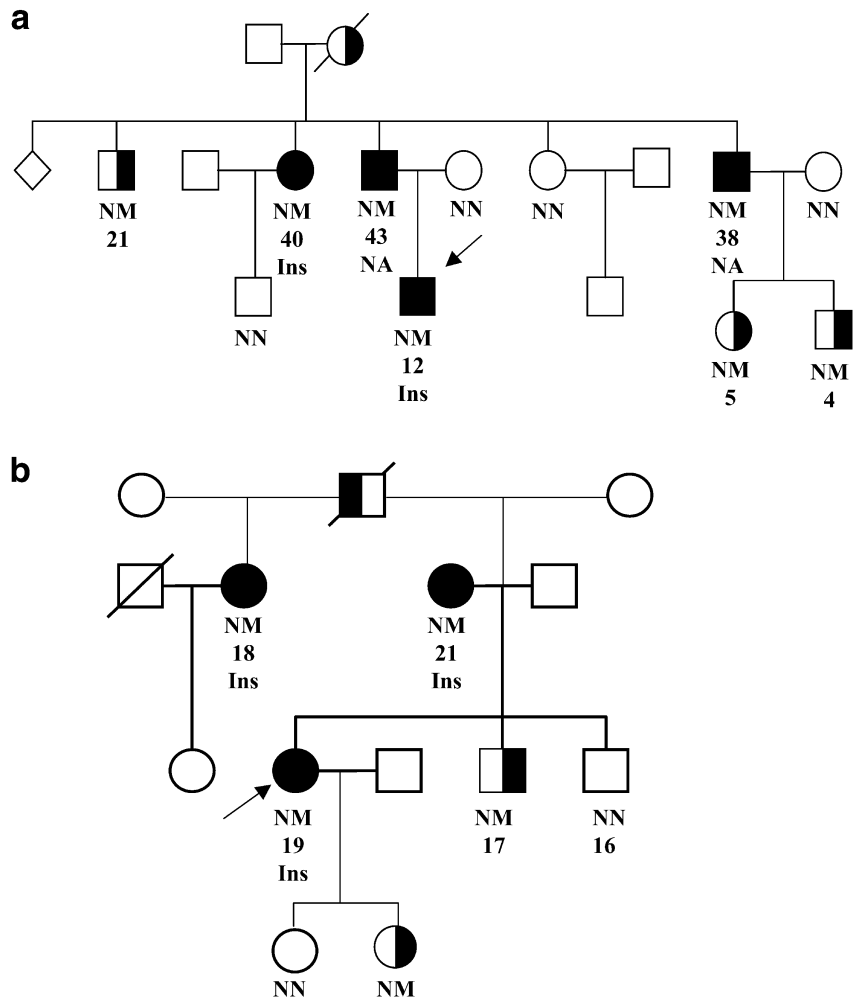

Fig. 1. Pedigrees for families in this study. a Family DUK504 (IVS2nt+1G>T). b Family DUK350 (IVS2nt+2insT). Individuals with renal disease are identified by a symbol with a filled right half. Individuals with diabetes are identified by a symbol with a filled left half. Age at diagnosis or examination and treatment for diabetes is shown (Ins, insulin; NA, not available). Where both phenotypes are present, the whole symbol is filled. The mutation status of each individual (where tested) is given ( $\mathrm{N}$, normal; $\mathrm{M}$, mutation). Both family histories are consistent with a diagnosis of renal cysts and diabetes syndrome. RNA was isolated from cell lines derived from the proband, marked with an arrow in each case

\section{Subjects, materials and methods}

Subjects. Two families were investigated in this study: family DUK504 (Fig. 1a) and family DUK350 (Fig. 1b).

Family DUK504 has previously been reported as having renal cysts, diabetes and atypical familial juvenile hyperuricaemic nephropathy [11]. In this family, the mutation IVS2nt $+1 \mathrm{G}>\mathrm{T}$ has already been described [11]. The proband developed diabetic ketoacidosis at the age of 12 and is treated with insulin. The proband's father developed diabetes at the age of 43, and a paternal uncle was diagnosed at age 38 (treatment unknown). A paternal aunt developed diabetes at the age of 40 and is treated with insulin.

In the previously unreported family DUK350, the proband developed diabetes at age 19 during her first pregnancy. She was treated with insulin during and after the pregnancy. During her second pregnancy, through an antenatal ultrasound scan at 27 weeks of gestation, her child was found to have renal cysts. This prompted scanning of the proband, where renal cysts were also detected. The proband's mother and maternal aunt developed diabetes aged 21 and 18 years respectively and are treated with insulin. They were both found to have renal cysts following an ultrasound screening. The maternal grandfather 
Table 1. Primers used for nested RT-PCR of HNF-1 $\beta$

\begin{tabular}{|c|c|}
\hline Primer set & Sense/antisense primer \\
\hline Round one & $\begin{array}{l}\text { Fragment 1-3a } \\
\text { 1B Ex1FP 5' atggtgtccaagctcacgtcg } 3^{\prime} \\
\text { 1B Ex3RP 5' gcattcctccactaaggcctctct } 3^{\prime} \\
\text { Fragment 3-6a } \\
\text { 1B Ex3FP 5' aaccagacagtccagagttctg } 3^{\prime} \\
\text { 1B Ex6RP 5' gactccagagaggggtgtcat } 3^{\prime} \\
\text { Fragment 5-8a } \\
\text { 1B Ex5FP 5' gagatcacttcctcctcaaca } 3^{\prime} \\
\text { 1B Ex8RP 5' acttgaagacatgttggtgag } 3^{\prime} \\
\text { Fragment 5-9a } \\
\text { 1B Ex5FP 5' gagatcacttcctcctcaaca } 3^{\prime} \\
\text { 1B Ex9RPS 5' tcaccaggcttgtagaggaca } 3^{\prime}\end{array}$ \\
\hline Round two & $\begin{array}{l}\text { Fragment } 1-3 \mathrm{~b} \\
\text { 1B Ex1FS 5' ctgagcgcctgctgagctcc } 3^{\prime} \\
\text { 1B Ex3RS 5' ccgatcgtaggcctggtacaa } 3^{\prime} \\
\text { Fragment 3-6b } \\
\text { 1B Ex3FS 5' agcagtcaggatcagctgctg } 3^{\prime} \\
\text { 1B Ex6RS 5' ctggggtaaatggtgggagag } 3^{\prime} \\
\text { Fragment 5-8b } \\
\text { 1B Ex5FS 5' agcgccatggtgaccagccag } 3^{\prime} \\
\text { 1B Ex8RS 5' ggtatctgtgaccaccattgc } 3^{\prime} \\
\text { Fragment 5-9b } \\
\text { 1B Ex5FS 5' agcgccatggtgaccagccag } 3^{\prime} \\
\text { 1B Ex9RPS 5' tcaccaggcttgtagaggaca } 3^{\prime}\end{array}$ \\
\hline
\end{tabular}

(now deceased) was also diabetic. Renal cysts were also detected in one of the proband's brothers. He had a normal oral glucose tolerance test at the age of 19 years. Renal function as assessed by serum creatinine showed mild renal impairment in all the affected family members other than the proband's brother, who had normal renal function. Hyperuricaemia was evident in all four adults.

Sequencing of the coding regions, intron/exon boundaries and minimal promoter of the $H N F-1 \beta$ gene was performed according to previously published methods [6]. The study was approved by the local ethics committee and informed consent was obtained from each subject.

Establishment of transformed lines. The cell lines ABO133 (unaffected control), WT0652 (proband DUK350) and MY0407 (pro- band DUK504) were established by Epstein-Barr virus (EBV) transformation of peripheral blood lymphocytes by the European Collection of Cell Cultures (Porton Down, Salisbury, UK).

Tissue culture and nonsense-mediated decay inhibitor experiments. Cell lines were maintained in 1× RPMI 1640 (Life technologies, Paisley, UK) supplemented with 10\% FCS (Life Technologies). Cycloheximide (an inhibitor of the NMD pathway) was added at $100 \mu \mathrm{g} / \mathrm{ml}$ in DMSO to investigate the possibility of NMD. A solvent control (DMSO 1\% v/v) was also tested.

Amplification of illegitimately transcribed mRNA from transformed lymphoblastoid cell lines. Total RNA was extracted from approximately $1 \times 10^{6} \mathrm{EBV}$-transformed lymphoblastoid cells using the Perfect RNA Mini RNA kit (Eppendorf, Hamburg, Germany) according to manufacturer's instructions. Complementary DNA (cDNA) was synthesised from $5 \mu \mathrm{g}$ of the total RNA using the Thermoscript RT-PCR system (Life Technologies) according to the manufacturer's instructions. The incubation temperature was $50{ }^{\circ} \mathrm{C}$. RT-PCR reactions were set up in triplicate in a tworound nested PCR with three overlapping fragments covering the entire $H N F-1 \beta$ cDNA from mutation-bearing cell lines and from unaffected controls (for primer sequences see Table 1). We used $2 \mu \mathrm{l}$ of each cDNA in each first-step PCR reaction. For the second round, $1 \mu \mathrm{l}$ of a 1:10 dilution was used. Conditions for both rounds of PCR were as follows: $3 \mathrm{~min}$ at $95{ }^{\circ} \mathrm{C}$ initial denaturation, followed by 35 cycles of $1 \mathrm{~min}$ at $95{ }^{\circ} \mathrm{C}, 1 \mathrm{~min}$ at $55^{\circ} \mathrm{C}$, $2 \mathrm{~min}$ at $72{ }^{\circ} \mathrm{C}$ and a final 10 -min extension at $72{ }^{\circ} \mathrm{C}$. We used 2.5 U of Taq polymerase (Abgene, Epsom, UK) per reaction.

RT-PCR products were run on $0.8 \%$ metaphor agarose gels (Helena Biosciences, Cambridge, UK). Individual splice products were isolated by removing small samples from the gels using a pipette tip and subsequent reamplification of DNA from the agarose gel plugs (bandstab PCR). PCR products were then purified (Qiagen PCR Purification Kit; Qiagen, Crawley, UK) and sequenced in both directions using Big Dye chemistry and an ABI 377 Sequencer (Applied Biosystems, Warrington, UK). Sequences were aligned with the published sequence using the Sequence Navigator software package (Applied Biosystems).

Real-time PCR quantification of splice variants. Ectopic mRNA transcripts were amplified from lymphoblastoid cells using a TaqMan approach. Splice variants purified by bandstab PCR as described above were used in serial dilution to produce standard curves for probe validation. Primer and probe sequences are given in Table 2. Three replicate reactions were

Table 2. Primers used for real-time PCR analysis of $H N F-1 \beta$ splice variants $\beta$

\begin{tabular}{|c|c|c|c|}
\hline \multicolumn{2}{|l|}{ Splice variant } & \multirow{2}{*}{$\begin{array}{l}\text { Sequence } \\
\text { 5' GTA CGT CAG AAA GCA ACG AGA GAT 3' } \\
\text { 5' TGA CTG CTT TTG TCT GTC ATA TTT CCA3' } \\
\text { 5' CCT CCG ACA ATT CAA C 3' }\end{array}$} & \multirow{2}{*}{ 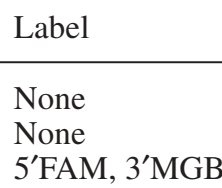 } \\
\hline Variant A & $\begin{array}{l}\text { F primer } \\
\text { R primer } \\
\text { Probe }\end{array}$ & & \\
\hline Variant B & $\begin{array}{l}\text { F primer } \\
\text { R primer } \\
\text { Probe }\end{array}$ & 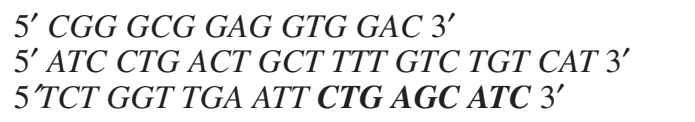 & $\begin{array}{l}\text { None } \\
\text { None } \\
\text { 5'FAM, 3'MGB }\end{array}$ \\
\hline Variant B deleted & $\begin{array}{l}\text { F primer } \\
\text { R primer } \\
\text { Probe }\end{array}$ & $\begin{array}{l}5^{\prime} \text { ACG TCA GAA AGC AAC GAG AGA TC 3' } \\
\text { 5' CCC AGG CCC ATG GCT 3' }^{\prime} \text { 5' TCC GAC AGT TCA GTC AAC A 3' }\end{array}$ & 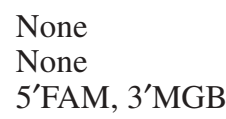 \\
\hline
\end{tabular}

Areas of probe located in different exons are marked in bold type. FAM, 6-fluoroscein; MGB, minor-groove-binding protein 
carried out for each cell line using $1.5 \mu \mathrm{l}$ cDNA. PCR products were detected using transcript-specific probes designed to span the exon-exon junctions (see Table 2). Reactions contained $10 \mu \mathrm{l}$ TaqMan Universal PCR Master Mix, no AmpErase, $1 \mu \mathrm{l}$ Assays-by-Design probe and primer mix (corresponding to $18 \mu \mathrm{mol} / \mathrm{l}$ per primer and $5 \mu \mathrm{mol} / \mathrm{l}$ per probe) in a total volume of $20 \mu \mathrm{l}$. Amplification conditions were a single cycle of $95^{\circ} \mathrm{C}$ for $10 \mathrm{~min}$, followed by 40 cycles of $95^{\circ} \mathrm{C}$ for 15 seconds and $60{ }^{\circ} \mathrm{C}$ for $1 \mathrm{~min}$.

Relative quantitation of $m R N A$ transcripts. Crossing points $(\mathrm{Ct})$ were determined for each splice variant and an endogenous control (the human $\beta$-actin gene). The relative abundance of each transcript was then determined from the equation $2^{-\Delta \Delta}$ Ct [20]. This equation relates to the difference in crossing point between each variant transcript and the $\beta$-actin endogenous control $\left(\Delta \mathrm{Ct}\right.$ test $\left.^{\text {te }}\right)$ relative to the $\Delta \mathrm{Ct}$ obtained from a reference sample $\left(\Delta \mathrm{Ct}^{\mathrm{ref}}\right) . \Delta \Delta \mathrm{Ct}$ is given by $\Delta \mathrm{Ct}^{\text {test }}-\Delta \mathrm{Ct}^{\mathrm{ref}}$. The levels of splice variant A were taken as the sample reference; other transcripts were therefore measured relative to wild-type transcript A. Once the proportion of each transcript in the mRNA population is known, the percentage of each isoform can be determined. This method gives an accurate measurement of relative abundance that is independent of all other factors.

\section{Results}

A novel splice site mutation, IVS2nt+2insT, was identified in the proband of family DUK350. The mutation co-segregated with renal disease and diabetes (Fig. 1b). This is the fourth mutation identified at the intron 2 donor splice site and confirms this as the $H N F-1 \beta$ mutation hot spot, representing 24\% (4/17) of mutations.

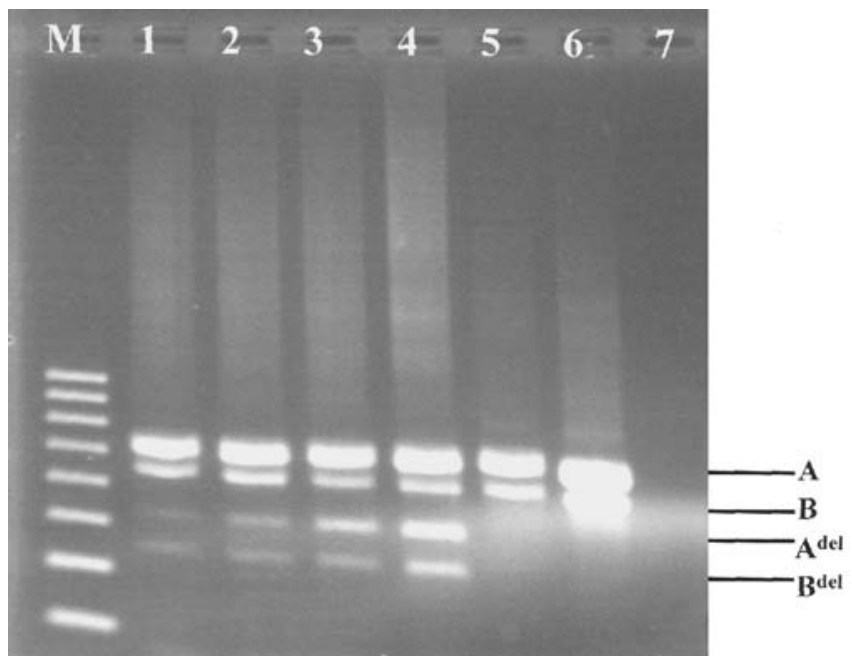

Fig. 2. Splice variants produced by the IVS $2+1 \mathrm{G}>\mathrm{T}$ and IVS2nt+2insT mutations. This figure shows the amplicons produced by RT-PCR of transcripts produced by IVS2nt+1G $>$ T mRNA for exons $1-4$. Size markers (100 bp ladder) are represented by 'M'. Lanes 1 and 2 are HNF-1 $\beta$ RT-PCR products amplified from proband DUK350. Lanes 3 and 4 are RT-PCR products amplified from the proband of family DUK504. Lanes 5 and 6 are RT-PCR products from unaffected controls. The water (negative) control is in lane 7. A and B are wildtype transcripts; $\mathrm{A}^{\mathrm{del}}$ and $\mathrm{B}^{\mathrm{del}}$ are mutated transcripts
Mutations at the splice donor site of intron 2 are predicted to lead to exon loss or retention, but in order to define the precise genetic defect, mRNA analysis was necessary. In the absence of samples from tissues with legitimate $H N F-1 \beta$ expression, we have used a novel RT-PCR assay to allow examination of $H N F-1 \beta$ mRNA from ectopic mRNA transcripts. We amplified the $H N F-1 \beta$ gene in three overlapping fragments from cell lines derived from the proband in each family and also from unrelated unaffected controls.

Agarose gel electrophoresis of the fragment amplifying exon 1 to exon 4 produced four products in both cell lines from affected individuals (Fig. 2), but only two products in the control subjects. Other fragments covering the remaining exons showed a single band in both controls and both probands. The two transcripts shared by affected subjects and controls were identified by direct sequencing as wild-type $H N F-1 \beta$ splice variants $A$ and $B$, which have been previously described [21]. The remaining two amplicons were identified as mutant forms of splice variants $\mathrm{A}$ and $\mathrm{B}$ in

a

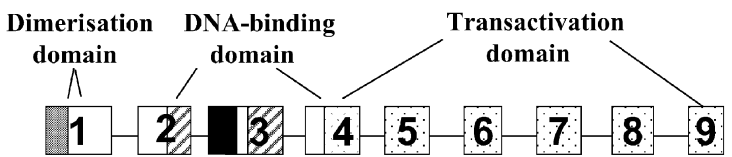

b

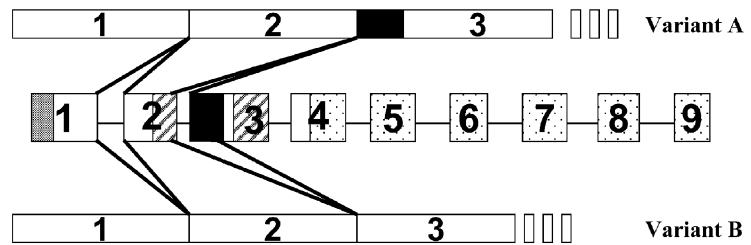

C

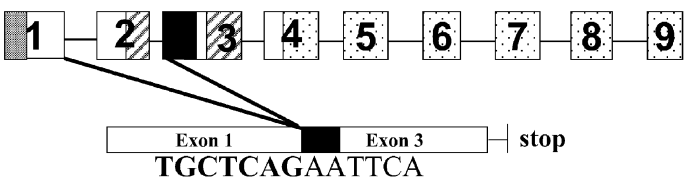

d

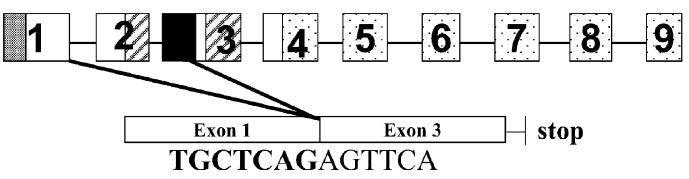

Fig. 3. a The functional domains of HNF-1 $\beta$. b Splicing pattern of wild-type variants A and B. c Splice variant Adel; exon 2 skipped, as frameshift leads to premature terminations at position codon 154. d Splice variant $\mathrm{B}^{\mathrm{del}}$; exon 2 skipped, as frameshift leads to premature terminations at position codon 128. The dimerisation domain located in exon 1 is shown in grey. The DNA binding domain (exons 2 and 3) is given by a hatched box. The transactivation domain (exons 4 to 9) is shown by a dotted box. The positions of the 26 amino acids spliced out to form variant B are shown in black. Schematics of mutant transcripts show the intron/exon structure of the relevant region of the $H N F-1 \beta$ gene, the predicted structure of the transcript following splicing and the true sequence of the junction region 
Table 3. Real-time quantitation of wild-type and abnormal $H N F-1 \beta$ splice variants

\begin{tabular}{llcclc}
\hline Mutation & Quantification & Variant A & Variant B & Variant A del & Variant Bdel \\
\hline Control & $\Delta \Delta \mathrm{Ct}$ & 0 & 0.67 & $\mathrm{n} / \mathrm{a}$ & $\mathrm{n} / \mathrm{a}$ \\
& & $61 \%$ & $39 \%$ & $\mathrm{n} / \mathrm{a}$ & $\mathrm{n} / \mathrm{a}$ \\
IVS2nt+1G>T -CHX & $\Delta \Delta \mathrm{Ct}$ & 0 & 1.15 & 2.93 & 3.86 \\
& & $61 \%$ & $27 \%$ & $8 \%$ & $4 \%$ \\
IVS2nt+1G>T +CHX & $\Delta \Delta \mathrm{Ct}$ & 0 & 1.54 & 2.78 & 2.56 \\
& & $60 \%$ & $21 \%$ & $9 \%$ & $10 \%$ \\
IVS2nt+2insT -CHX & $\Delta \Delta \mathrm{Ct}$ & 0 & 0.62 & 3.82 & 2.66 \\
IVS2nt+2insT +CHX & $\Delta \Delta \mathrm{Ct}$ & $53 \%$ & $35 \%$ & $4 \%$ & $8 \%$ \\
& & 0 & 0.62 & 3.39 & 2.07 \\
\hline
\end{tabular}

n/a, not applicable

which exon 2 was deleted (Adel, Bdel; Fig. $3 \mathrm{~b}$ and $3 \mathrm{c}$ ). We were unable to detect $H N F-1 \beta$ splice variant $\mathrm{C}$ in any sample, perhaps due to a low level of expression, which prevents amplification from ectopic transcripts. The predicted effect of deleting exon 2 would be the generation of a frameshift leading to a premature termination at codon 154 for splice variant $\mathrm{A}$ and at codon 128 for splice variant B. The effect of such a change would be the disruption of the DNA-binding domain of the protein and the complete deletion of the transactivation domain. Such a protein is likely to have little or no activity.

The abnormal exon 1-4 amplicons from the cell lines with $H N F-1 \beta$ mutations showed reduced intensity on agarose gel electrophoresis compared with the wild-type products (Fig. 2). The relative levels of each transcript were then quantified by real-time PCR. In the control cell line, the relative levels of splice variants $\mathrm{A}$ and $\mathrm{B}$ were estimated at $61 \%$ and $39 \%$ respectively (Table 3). Cells harbouring the IVS2nt+1G $>$ T mutation had $H N F-1 \beta$ transcript levels of $61 \%, 27 \%$, $8 \%$ and $4 \%$ for transcripts $\mathrm{A}, \mathrm{B}, \mathrm{A}^{\mathrm{del}}$ and $\mathrm{B}^{\mathrm{del}}$ respectively (see Table 3). The mutation IVS2nt+2delT showed transcript frequencies of $53 \%, 35 \%, 4 \%$ and $8 \%$ for transcripts $\mathrm{A}, \mathrm{B}, \mathrm{A}^{\mathrm{del}}$ and $\mathrm{B}^{\mathrm{del}}$ respectively.

Since both mutant transcripts generate frameshift mutations leading to the production of premature termination codons, they are theoretically substrates for the NMD mRNA surveillance pathway. We therefore sought to determine whether the lower abundance of mutant transcripts was due to a down-regulation of transcription or to the action of NMD by the use of the NMD inhibitor cycloheximide. The frequency of isoforms present in cycloheximide-treated IVS2nt $+1 \mathrm{G}>\mathrm{T}$ mRNA were $60 \%, 21 \% 9 \%$ and $10 \%$ respectively (Table 3). The frequency of wild-type and abnormal transcripts produced from the IVS2nt+2delT mutation were $50 \%, 33 \%, 5 \%$ and $12 \%$ respectively (Table 3 ). The translation inhibitor cycloheximide did not influence the ratio of abnormal to normal transcripts in cells carrying either mutation: $19 \%$ vs $23 \%$ and $14 \%$ vs $20 \%$ for the IVS2nt+1G>T and IVS2nt+2delT mutations respectively. This suggests that the lower relative level of mutant splice variants is probably not due to NMD.

\section{Discussion}

The most common site for diabetes-associated mutations in the $H N F-1 \beta$ gene is the splice donor site of intron 2. We report a novel mutation, IVS2nt+2insT, the fourth mutation at this site. This paper describes the first $H N F-1 \beta$ splice site mutations to be studied at the mRNA level from subjects with the RCAD syndrome. Both mutations generate identical transcripts with abnormal splicing and deletion of exon 2 , which is predicted to result in a truncated protein lacking the transactivation domain. The affected members of families DUK350 and DUK504 who carry the splice site mutations have similar phenotypes, and it is perhaps not surprising that both mutations are predicted to operate by the same mechanisms. In both families there are affected subjects with renal cysts and early-onset diabetes, the cardinal features of the RCAD syndrome. Family DUK504 has previously been reported as having hyperuricaemia and gout, but these are features that have recently been described in association with many $H N F-1 \beta$ mutations [11]. All the affected adult subjects in family DUK350 also have hyperuricaemia. Other families with mutations in this region also report renal cysts (without defined histology) and diabetes $[9,11,14]$. It is likely therefore that all families with mutations at this site have a similar phenotype, although there can be variation between family members, as seen in both our families (Fig. 1).

Using real-time PCR, we showed that the mutated splice variants are present at a lower level than the native transcripts. Mutant transcripts containing premature termination codons may be detected and degraded by NMD, a ubiquitous process in eukaryotic cells. NMD has previously been reported to be a result of 
splice site mutations [16]. However, the inclusion of the NMD inhibitor, cycloheximide, failed to alter the relative transcript frequencies as measured by realtime PCR. This suggests that the reduction of the mutant transcript frequency is unlikely to be due to NMD. It is possible that the mutated mRNAs represent minor splice products, but it is more likely that their stability is compromised by other mechanisms. Mutations that induce conformational changes in the tertiary structure of dopamine receptor mRNA transcripts have recently been demonstrated to decrease the stability of the transcripts in vivo [22].

The presence of ectopic transcripts in lymphoblastoid cell lines has allowed examination of mRNA splicing mechanisms in human genes where studies of mRNA expression are limited by the expression profile. Using this technology we have been able to demonstrate the effects of two $H N F-1 \beta$ mutations, IVS2nt+2insT and IVS2nt+1G $>$ T, on RNA splicing. The consequences of mutations that affect mRNA processing are difficult to predict from the genomic DNA structure alone. It is therefore important to conduct studies at the mRNA level. This type of analysis may elucidate the molecular mechanisms of splice site mutations and aid confirmation of their pathogenic status. Although we believe that the analysis of ectopic transcripts provides a good model for tissues with legitimate $H N F-1 \beta$ expression, there can be no final proof of this until such tissues become available.

Acknowledgements. We are grateful to the Wellcome Trust, Diabetes UK, the National Kidney Research Fund (grant TF13/ 2000), the European Union (contract number QLG-CT-199900546 [GIFT]) and the Royal Devon \& Exeter NHS Healthcare Trust R\&D Directorate for financial support. A. T. Hattersley is a Wellcome Trust research leave fellow and C. Bingham is an NKRF clinical research fellow. The European Collection of Cell Cultures (ECACC) kindly provided the cell lines. The authors are grateful to W. G. Van't Hoff for the referral and for providing the clinical details for family DUK504.

\section{References}

1. Horikawa Y, Iwasaki N, Hara M et al. (1997) Mutation in hepatocyte nuclear factor-1 beta gene (TCF2) associated with MODY. Nat Genet 17:384-385

2. Nishigori H, Yamada S, Kohama T et al. (1998) Frameshift mutation, A263fsinsGG, in the hepatocyte nuclear factor-1 beta gene associated with diabetes and renal dysfunction. Diabetes 47:1354-1355

3. Bingham C, Bulman M, Ellard S et al. (2001) Mutations in the HNF-1beta gene are associated with familial hypoplastic glomerulocystic kidney disease. Am J Hum Genet 68: 219-224

4. Bingham C, Ellard S, Cole TR et al. (2002) Solitary functioning kidney and diverse genital tract malformations associated with hepatocyte nuclear factor-1 beta mutations. Kidney Int 61:1243-1251

5. Kolatsi-Joannou M, Bingham C, Ellard S et al. (2001) Hepatocyte nuclear factor-1beta: a new kindred with renal cysts and diabetes and gene expression in normal human development. J Am Soc Nephrol 12:2175-2180

6. Bingham C, Ellard S, Allen L et al. (2000) Abnormal nephron development associated with a frameshift mutation in the transcription factor hepatocyte nuclear factor-1 beta. Kidney Int 57:898-907

7. Lindner TH, Njolstad PR, Horikawa Y, Bostad L, Bell GI, Sovik O (1999) A novel syndrome of diabetes mellitus, renal dysfunction and genital malformation associated with a partial deletion of the pseudo-POU domain of hepatocyte nuclear factor-1beta. Hum Mol Genet 8:2001-2008

8. Mache CJ, Preisegger KH, Kopp S, Ratschek M, Ring E (2002) De novo HNF-1beta gene mutation in familial hypoplastic glomerulocystic kidney disease. Pediatr Nephrol 17:1021-1026

9. Iwasaki N, Okabe I, Momoi MY, Ohashi H, Ogata M, Iwamoto Y (2001) Splice site mutation in the hepatocyte nuclear factor-1 beta gene, IVS2nt $+1 \mathrm{G}>\mathrm{A}$, associated with maturity-onset diabetes of the young, renal dysplasia and bicornuate uterus. Diabetologia 44:387-388

10. Montoli A, Colussi G, Massa O et al. (2002) Renal cysts and diabetes syndrome linked to mutations of the hepatocyte nuclear factor- 1 beta gene: description of a new family with associated liver involvement. Am J Kidney Dis 40:397-402

11. Bingham C, Ellard S, Van't Hoff WG et al. (2003) Atypical familial juvenile hyperuricemic nephropathy associated with a hepatocyte nuclear factor-1beta gene mutation. Kidney Int 63:1645-1651

12. Owen K, Hattersley AT (2001) Maturity-onset diabetes of the young: from clinical description to molecular genetic characterization. Best Pract Res Clin Endocrinol Metab 15:309-323

13. Iwasaki N, Ogata M, Tomonaga O et al. (1998) Liver and kidney function in Japanese patients with maturity-onset diabetes of the young. Diabetes Care 21:2144-2148

14. Carbone I, Cotellessa M, Barella C et al. (2002) A novel hepatocyte nuclear factor-1beta (MODY-5) gene mutation in an Italian family with renal dysfunctions and early-onset diabetes. Diabetologia 45:153-154

15. Furuta H, Furuta M, Sanke T et al. (2002) Nonsense and missense mutations in the human hepatocyte nuclear factor- 1 beta gene (TCF2) and their relation to type 2 diabetes in Japanese. J Clin Endocrinol Metab 87:3859-3863

16. Frischmeyer PA, Dietz HC (1999) Nonsense-mediated mRNA decay in health and disease. Hum Mol Genet 8:1893-1900

17. Byers PH (2002) Killing the messenger: new insights into nonsense-mediated mRNA decay. J Clin Invest 109:3-6

18. Bulman MP, Harries LW, Hansen T et al. (2002) Abnormal splicing of hepatocyte nuclear factor 1 alpha in maturityonset diabetes of the young. Diabetologia 45:1463-1467

19. Sun F, Knebelmann B, Pueyo ME et al. (1993) Deletion of the donor splice site of intron 4 in the glucokinase gene causes maturity-onset diabetes of the young. J Clin Invest 92:1174-1180

20. Applied Biosystems (2001) Relative quantitation of gene expression. User bulletin no. 2:11-15

21. Bach I, Yaniv M (1993) More potent transcriptional activators or a transdominant inhibitor of the HNF1 homeoprotein family are generated by alternative RNA processing. EMBO J 12:4229-4242

22. Duan J, Wainwright MS, Comeron JM et al. (2003) Synonymous mutations in the human dopamine receptor D2 (DRD2) affect mRNA stability and synthesis of the receptor. Hum Mol Genet 12:205-216 